\title{
Traduire
}

Revue française de la traduction

$239 \mid 2018$

Traduire l'art

\section{Retracer l'acte de création : Les Niaoulis (1966) de Nicole Vedrès}

Jérôme Allain

\section{CpenEdition}

Journals

Édition électronique

URL : http://journals.openedition.org/traduire/1534

DOI : 10.4000/traduire.1534

ISSN : 2272-9992

\section{Éditeur}

Société française des traducteurs

\section{Édition imprimée}

Date de publication : 1 décembre 2018

Pagination : 47-52

ISSN : 0395-773X

\section{Référence électronique}

Jérôme Allain, «Retracer l'acte de création : Les Niaoulis (1966) de Nicole Vedrès », Traduire [En ligne], 239 | 2018, mis en ligne le 01 décembre 2018, consulté le 29 juin 2019. URL : http://

journals.openedition.org/traduire/1534 ; DOI : 10.4000/traduire.1534 


\section{Retracer l'acte de création : Les Niaoulis (1966) de Nicole Vedrès}

\section{Jérôme Allain}

Depuis la publication en 1943 de son premier ouvrage, Un siècle d'élégance française, suivi en 1945 du Théâtre de la mode en collaboration avec Thierry Maulnier et Jacques Wilhelm, l'auteure, réalisatrice et chroniqueuse française Nicole Vedrès (1911-1965) s'est toujours gardée de parler de son œuvre lors d'émissions de radio ou d'entretiens accordés à la presse généraliste et spécialisée. La rareté de ses documents de travail et donc l'absence de fonds d'archives sont autant de facteurs qui rendent difficile l'exploration du chercheur, qui ne peut pas s'appuyer sur des éléments probants pour reconstruire la pratique ou la réflexion menée par l'auteure lors du processus de création. Aussi, lorsqu'en mai 1961, Georges Piroué, journaliste pour la revue Le Mercure de France, aborde au cours d'un entretien la question du travail de l'artiste face à son œuvre, Nicole Vedrès lui répond :

Qu'est-ce que vous voulez que le peintre réponde si on lui demande : "Pourquoi ce rouge? "II n'en sait rien. Pas plus que le coureur de jupons ne sait pourquoi il court. Vous vous voyez exiger de lui un traité d'érotologie sous prétexte qu'il fait bien l'amour!

Toutefois, l'existence de documents administratifs (bons de commande, factures, notes) émanant de différents fonds d'archives publiques ou privées permet d'appréhender le regard de cette auteure sur ses œuvres, voire sur sa pratique. La difficulté pour le chercheur est alors de parvenir à traduire, à interpréter et à identifier un ensemble de données contenues dans les documents et pièces d'archives. C'est au cours de recherches consacrées à Nicole Vedrès et à sa pièce de théâtre Les Canaques que j'ai été amené à me questionner en tant qu'archiviste sur l'usage des bordereaux, guides, inventaires et répertoires consultables dans les services d'archives et permettant d'accéder aux fonds répertoriés et classés. Nous relevons l'objet de son sujet, la cote qui lui est associée afin de consulter l'article souhaité puis refermons l'instrument de recherche. Mais est-ce le seul usage de ces documents ? N'y aurait-il pas une autre interprétation?

Nicole Vedrès a réalisé Paris 1900, un film de montage sorti en 1947. Elle a également écrit plusieurs ouvrages tels que Les Cordes rouges ou encore La fin de septembre, publiés 
respectivement en 1953 et en 1962. Elle est davantage connue en tant que chroniqueuse pour le journal Le Mercure de France, dans l'émission télévisée Lectures pour tous ainsi que dans l'émission radiophonique Le Masque et la plume. Bien qu'elle soit considérée comme l'une des figures importantes de l'après-guerre dans le quartier Saint-Germain-des-Prés, il n'existe aucun fonds d'archives dont elle serait productrice ou auteure. II a donc fallu recourir à d'autres fonds afin de relever des documents mentionnant son nom et, d'une certaine façon, traduire le processus créatif de l'auteure, afin de parvenir à reconstituer avec minutie son œurre et d'en comprendre les tenants et aboutissants à travers des manques épars.

Au cours de l'année 2013, ces recherches se sont orientées sur l'unique pièce de théâtre de Nicole Vedrès, Les Canaques, ayant fait l'objet d'une publication en 1966 aux Éditions du Seuil. Ce titre n'est cité que dans deux documents d'archives: l'une des fiches des auteurs qui ont collaboré avec les éditions du Seuil, sur laquelle est noté "Théâtre à paraître aux Éditions du Seuil. Les Canaques, février 1966 " ; et une lettre datée du 4 août 1972 de François-Régis Bastide, conseiller littéraire aux éditions du Seuil à partir de 1953, dans laquelle il évoque une possible adaptation à la télévision de la pièce de théâtre de Nicole Vedrès. Mais aucun de ces documents ne s'attache à décrire d'une quelconque façon la pièce. Si d'autres œuvres de Nicole Vedrès ont fait l'objet de correspondances professionnelles et privées, notamment avec sa mère, et ont permis d'entrevoir leur genèse, comment une pièce de théâtre parue et vraisemblablement mise en scène en France, pourrait ne pas être citée, commentée et présentée par l'éditeur lui-même ou son auteur ? Certaines archives de l'auteur n'ont peut-être pas été déposées ou pire, ont disparu. Pourtant, sa pièce de théâtre intitulée Les Canaques a donné lieu à un discours dont l'interprétation a permis de mieux appréhender une partie du processus créatif de l'auteure. L'ouvrage publié en 1966 nous indique que l'intrigue des Canaques se déroule en Nouvelle-Calédonie aux débuts des années 1860. S'y croisent différents personnages : missionnaire, déporté, savant, officier français, bagnard, cantatrice, voyageur, etc. Au centre de cette faune émerge la figure d'Arthur, un aventurier solitaire. Son prénom retient l'attention puisque le premier ouvrage littéraire de Nicole Vedrès s'intitule Le labyrinthe ou le jardin de Sir Arthur. Mais un autre fait est à noter : dans l'épisode "Démons et merveilles du cinéma " du 21 novembre 1964 qui lui est consacré, Nicole Vedrès raconte qu'à l'âge de cinq ans, elle est tombée amoureuse de son oncle Arthur. Cet homme, né selon elle dans les années 1850, a été exilé par sa famille en Australie vers 1870. Cet événement familial a probablement inspiré la pièce de théâtre Les Niaoulis, dont la version finale deviendra l'ouvrage Les Canaques. Un document interne de la maison d'édition du Seuil tend à confirmer cette hypothèse. En octobre 1964, François-Régis Bastide rédige un " schéma d'analyse " d'un projet littéraire de Nicole Vedrès intitulé Les Niaoulis. Cette pièce de théâtre lui évoque "une mise en scène de Planchon, pleine de couleurs et de mouvement ". Le croisement des informations relevées dans ces documents fait apparaître les points suivants : 


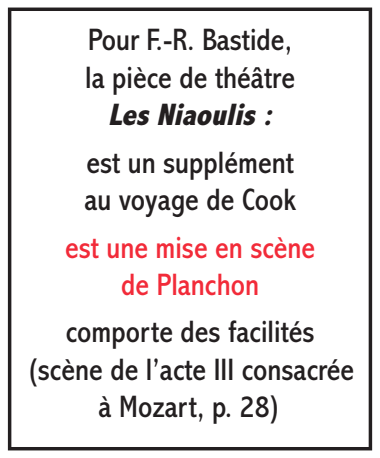
L'ouvrage Les Canaques
relate une histoire :
celle d'Arthur,
le personnage principal
où se croisent des alsaciens
et des bagnards
qui évoque Mozart
(scène de l'acte III, p. 121)
qui se déroule en Nouvelle-
Calédonie vers 1870

Pour Nicole Vedrès, la pièce de théâtre Les Niaoulis :

peut se rapprocher du Printemps 71 d'Adamov met en scène son oncle Artur, le personnage principal met en scène des alsaciens, des bagnards et des communards

Les Niaoulis et Les Canaques sont bien une seule et même œuvre. Les documents de travail consultés dans différents fonds d'archives répondent donc au schéma d'acte de création énoncé au début de cet article : l'opportunité pour le chercheur de comprendre la genèse d'une œuvre en interprétant les manques et absences dans la correspondance privée de l'auteur.

Pourtant, moins de $50 \%$ du potentiel de l'instrument de recherche a été exploité au cours de cette recherche, soit quatre sections sur les neuf disponibles. II est apparu intéressant de s'interroger sur l'utilisation des instruments de recherche mis à la disposition des usagers. Si le peu d'informations ou l'absence de documents d'archives sur le sujet questionné par le chercheur pourrait l'inciter à arrêter ses travaux, n'est-ce pas le chercheur lui-même qui restreint sa recherche par sa façon de questionner le répertoire lui présentant les documents susceptibles de répondre à sa quête ? Ne faudrait-il pas recourir à l'instrument de recherche non plus comme un simple outil d'accès, mais en l'interrogeant comme n'importe quel document d'archives?

II s'agit ici d'aborder le répertoire(1) à la manière d'une source à exploiter, interpréter et traduire de façon à fonder des hypothèses ou des pistes de réflexion. Le répertoire n'est, dans le cas qui nous intéresse, ni une source par substitution, ni un outil méthodologique comme le guide de recherche. En d'autres termes, nous allons tenter, en nous appuyant sur la partie introductive du répertoire ainsi que sur le plan de classement, de démontrer qu'il est possible d'extraire des informations d'un instrument de recherche sans pour autant consulter les archives répertoriées auxquelles il renvoie. Cet emploi du répertoire comme source par destination est envisageable pour trois raisons.

(1) Un répertoire se compose d'une introduction - comptant sept zones de description qui répondent à la norme internationale $\operatorname{ISAD}(G)$ - et d'un plan de classement. 
Premièrement, il existe un discours portant sur le répertoire comme source. En avril 2010, Christine Nougaret(2) rappelle dans le numéro 220 de La Gazette des archives qu'en cas de " disparition " de documents d'archives, l'instrument de recherche conçu par l'archiviste peut être utilisé par le chercheur au même titre qu'une source. S'il est question ici des catalogues, guides, inventaires, registres et répertoires conçus par les services d'archives, il est nécessaire de rappeler que cette problématique a auparavant été traitée similairement par Michèle Lagny dans son ouvrage De l'histoire du cinéma. Professeur émérite d'histoire de la culture, elle préconise l'utilisation de " sources directes : catalogues de vente des maisons de production et de distribution, dont on retrouve des traces (souvent lacunaires) dans les archives, $[\ldots]^{(3)}$ " et ajoute " on trouvera ces instruments, par exemple en France, Le catalogue de la production cinématographique, publié par le CNC et le Service des Archives du film [... [(4) ॥. II s'agit bien dans ce cas précis d'utiliser ces outils en tant que source témoignant par exemple de l'activité d'un producteur, mais aussi permettant de travailler sur une histoire des mentalités. Ces deux discours démontrent qu'il est concevable de recourir au répertoire comme source par destination.

La deuxième raison qui permettrait au chercheur d'utiliser le répertoire comme source concerne les normes. En effet, l'instrument de recherche, et plus particulièrement son plan de classement, s'attache à refléter l'activité du producteur. Sa rédaction s'appuie sur la norme $\operatorname{ISAD}(G)$ adoptée en 1994, qui présente avec précision la manière dont l'archiviste doit établir la structure d'un répertoire à l'usage du lecteur, et sur l'instruction du 13 juin 2005, qui définit une liste de recommandations pour la rédaction du plan de classement d'un répertoire. Le répertoire utilisé comme source première est alors un "nouveau témoin " (expression empruntée à Michèle Lagny) puisque la norme et l'instruction définissent les conditions pour préserver l'intégrité de l'instrument de recherche, lui donnant par conséquent une valeur de preuve.

Enfin, la troisième et dernière raison d'envisager le répertoire comme une source par destination concerne ses limites. En effet, quel que soit le niveau de description d'un document d'archives, la principale difficulté de l'archiviste lorsqu'il traite un fonds est de relever quels noms ou quels mots feront l'objet d'une demande dans un futur plus ou moins proche. Ainsi, la rédaction d'un répertoire implique nécessairement l'apparition ou la conservation de nondits déjà présents dans les documents d'archives. Marie-Anne Chabin définit dans son ouvrage Je pense donc j'archive trois catégories de non-dit. La première, le " non-dit par négligence ",

(2) Conservateur général du patrimoine du Centre historique des Archives nationales, Christine Nougaret est une des spécialistes de la question des instruments de recherche. Elle a également participé à l'élaboration de la norme $\operatorname{ISAD}(G)$.

(3) LAGNY Michèle, p. 244.

(4) Ibid., p. 245. Le Centre national du cinématographe et de l'animé (CNC) a signé en 1977 une convention avec la Direction des Archives de France (aujourd'hui Service interministériel des archives de France, SIAF) pour recevoir le dépôt légal des œuvres cinématographiques. 
renvoie à l'absence de date, à la non-identification d'un destinataire, etc. La deuxième se rapporte aux " oublis volontaires ou intentionnés " : un répertoire ne peut être exhaustif ; sans se méprendre sur l'activité intellectuelle du producteur, celui-ci est amené à faire des choix. La troisième et dernière catégorie porte sur les "indices formels ", et invite à ne pas effacer la forme au profit du fond: on s'interrogera par exemple sur les implications d'une lettre adressée à un journal national qui n'aurait pas été envoyée et que le chercheur retrouve dans les archives de son auteur.

Je serais tenté d'ajouter une quatrième catégorie, que j'appelle le non-dit " externe ». Celui-ci n'est pas lié, comme les précédents, aux documents inventoriés par l'instrument de recherche, mais bien à l'instrument lui-même, et concerne les manques du répertoire. Si, pour l'archiviste, le répertoire contient une série d'informations qu'il a soigneusement rédigée, pour le chercheur, le répertoire est une source qui contient une série de données(5) qu'il lui faut exploiter.

Cette méthode offrirait au chercheur l'opportunité de porter un autre regard sur l'œuvre d'un auteur, tout en sachant qu'il devra consulter ces archives. D'une part, près de $75 \%$ du potentiel de l'instrument de recherche serait désormais exploité, soit sept sections sur les neuf disponibles. D'autre part, cette démarche permettrait au chercheur de prendre et de conserver une distance avec le sujet de sa recherche, aussi bien sur la question de l'intimité (la démarche créatrice de l'auteur) que sur celle de l'activité (la démarche productive de l'auteur).

Voilà comment, à partir des Canaques de Nicole Vedrès, j'ai tenté de mettre en évidence une double implication dans le traitement des fonds d'auteurs qui amène une double interprétation. Tout d'abord, en rappelant ce qui est reconnu comme une évidence : conserver les travaux intermédiaires, incomplets, abandonnés, utilisés comme brouillon par l'auteur permet au chercheur de mieux comprendre un acte de création; de mieux comprendre la genèse d'une œuvre grâce à la biographie et aux sources complémentaires du répertoire. C'est ainsi que les archivistes contribuent à la valorisation des arts par la rédaction des instruments de recherche. Enfin, le répertoire comme source par destination donne l'opportunité au chercheur de poursuivre son investigation par l'identification de nouvelles hypothèses ou pistes de réflexion sans consulter les documents inventoriés dans le plan de classement.

allainj4@gmail.com

(5) Je reprends les termes de J.-P. Pourtois, H. Desmet et W. Lahaye, de la Faculté de Psychologie et des Sciences de l'Éducation à l'Université de Mons-Hainaut, dans un cours portant sur la méthodologie de recherche en sciences appliquées. 
Ancien archiviste, diplômé des universités d'Angers et de Rennes 2, Jérôme Allain, agent dans la fonction publique territoriale, poursuit depuis 2013 des travaux autour de deux axes : le statut des instruments de recherche conçus pour les usagers des services d'archives, ainsi que les pratiques et recherches recourant aux archives publiques ou privées face à la masse d'informations et l'absence de données. Depuis 2015, il est également l'auteur de la chronique Art'chives dans la revue Archivistes! publiée par l'Association des Archivistes Français. En parallèle, il publie un carnet de recherche qui retrace l'œuvre de Nicole Vedrès, auteure, chroniqueuse, essayiste et réalisatrice française méconnue.

\section{Bibliographie}

ALLAIN Jérôme, 2014 [version enrichie en 2017], "Sur la trace de Nicole Vedrès ", https://paris1900.hypotheses.org, consulté le 18/02/2018.

ASSOCIATION DES ARCHIVISTES FRANÇAIS, DIRECTION DES ARCHIVES DE FRANCE, 1970, Manuel d'archivistique. Théorie et pratique des archives en France, Paris, Archives nationales. ASSOCIATION DES ARCHIVISTES FRANÇAIS, 1990-1991, La Gazette des archives [Les instruments de recherche aujourd'hui], 152-153.

ASSOCIATION DES ARCHIVISTES FRANÇAIS, 2010, La Gazette des archives [Les instruments de recherche : évolutions, publics et stratégies], 220.

CHABIN Marie-Anne, 1999, Je pense donc j'archive, l'archive dans la société d'information, Paris, L'Harmattan.

CORBIN Alain, 1998, Le monde retrouvé de François Pinagot, Paris, Flammarion.

FAVIER Jean et NEIRINCK Danièle, 1993, La pratique archivistique française, Paris, Archives nationales.

FOUCAULT Michel, 2008, L'archéologie du savoir, Paris, Gallimard, coll. "Tel ».

LAGNY Michèle, 1992, De l'histoire du cinéma, Paris, Armand Colin, coll. "Cinéma et audiovisuel".

NOUGARET Christine et GALLAND Bruno, 1999, Les instruments de recherche dans les archives, Paris, La documentation française et la Direction des archives de France.

POURTOIS Jean-Pierre, DESMET Huguette et LAHAYE Willy, 2013, Les points-charnières de la recherche scientifique, Faculté de Psychologie et des Sciences de l'Éducation, Université de Mons-Hainaut, https://mtcmadagascar.files.wordpress.com/2013/07/methodologie m2r.pdf, consulté le 12 août 2018.

VEDRÈS Nicole, 1966, Les Canaques, Paris, Mercure de France. 